Pacific Journal of Mathematic 


\title{
CONSTRUCTIVE VERSIONS OF TARSKI'S FIXED POINT THEOREMS
}

\author{
Patrick Cousot and RAdHIa Cousot
}

Let $F$ be a monotone operator on the complete lattice $L$ into itself. Tarski's lattice theoretical fixed point theorem states that the set of fixed points of $F$ is a nonempty complete lattice for the ordering of $L$. We give a constructive proof of this theorem showing that the set of fixed points of $F$ is the image of $L$ by a lower and an upper preclosure operator. These preclosure operators are the composition of lower and upper closure operators which are defined by means of limits of stationary transfinite iteration sequences for $F$. In the same way we give a constructive characterization of the set of common fixed points of a family of commuting operators. Finally we examine some consequences of additional semicontinuity hypotheses.

1. Introduction. Let $L(\subseteq, \perp, T, \cup, \cap)$ be a nonempty complete lattice with partial ordering $\subseteq$, least upper bound $U$, greatest lower bound $\cap$. The infimum $\perp$ of $L$ is $\cap L$, the supremum $T$ of $L$ is $U L$. (Birkhoff's standard reference book [3] provides the necessary background material.) Set inclusion, union and intersection are respectively denoted by $\subseteq, \underline{U}$ and $\cap$.

Let $F$ be a monotone operator on $L(\subseteq, \perp, T, \cup, \cap)$ into itself (i.e., $\forall X, Y \in L,\{X \subseteq Y\} \Longrightarrow\{F(X) \subseteq F(Y)\})$.

The fundamental theorem of Tarski [19] states that the set $f p(F)$ of fixed points of $F$ (i.e., $f p(F)=\{X \in L: X=F(X)\}$ ) is a nonempty complete lattice with ordering $\subseteq$. The proof of this theorem is based on the definition of the least fixed point $l f p(F)$ of $F$ by $l f p(F)=$ $\cap\{X \in L: F(X) \subseteq X\}$. The least upper bound of $S \subseteq f p(F)$ in $f p(F)$ is the least fixed point of the restriction of $F$ to the complete lattice $\{X \in L:(\cup S) \subseteq X\}$. An application of the duality principle completes the proof.

This definition is not constructive and many applications of Tarski's theorem (specially in computer science (Cousot [5]) and numerical analysis (Amann [2])) use the alternative characterization of $l f p(F)$ as $\cup\left\{F^{i}(\perp): i \in N\right\}$. This iteration scheme which originates from Kleene [10]'s first recursion theorem and which was used by Tarski [19] for complete morphisms, has the drawback to require the additional assumption that $F$ is semi-continuous $(F(\cup S)=\cup F(S)$ for every increasing nonempty chain $S$, see e.g., Kolodner [11]). 
The purpose of this paper is to give a constructive proof of Tarski's theorem without using the continuity hypothesis. The set of fixed points of $F$ is shown to be the image of $L$ by preclosure operations defined by means of limits of stationary transfinite iteration sequences. Then the set of common fixed points of a family of commuting monotone operators on a complete lattice into itself is characterized in the same way. The advantage of characterizing fixed points by iterative schemes is that they lead to practical computation or approximation procedures. Also the definition of fixed points as limits of stationary iteration sequences allows the use of transfinite induction for proving properties of these fixed points.

Finally some consequences of the additional and less general continuity hypothesis are examined.

\section{Definitions.}

DeFINITION 2.1. (Upper iteration sequence.) Let $L(\subseteq, \perp, T, \cup, \cap)$ be a complete lattice, $\mu$ the smallest ordinal such that the class $\{\delta: \delta \in \mu\}$ has a cardinality greater than the cardinality Card $(L)$ of $L$ and $F$ a monotone operator on $L$ into itself. The $\mu$-termed upper iteration sequence for $F$ starting with $D \in L$ is the $\mu$-termed sequence $\left\langle X^{o}, \delta \in \mu\right\rangle$ of elements of $L$ defined by transfinite recursion in the following way:

(a) $X^{0}=D$

(b) $X^{\delta}=F\left(X^{i-1}\right)$ for every successor ordinal $\delta \in \mu$

(c) $X^{\hat{o}}=\mathbf{U}_{\alpha<\delta} X^{\alpha}$ for every limit ordinal $\delta \in \mu$

(the dual lower iteration sequence is defined by:

( $\left.\mathrm{c}^{\prime}\right) \quad X^{\delta}=\bigcap_{\alpha<\delta} X^{\alpha}$ for every limit ordinal $\left.\delta \in \mu\right)$.

DEFINITION 2.2. (Limit of a stationary transfinite sequence.) We say that the sequence $\left\langle X^{o}, \delta \in \mu\right\rangle$ is stationary if and only if $\left\{\exists \varepsilon \in \mu:\left\{\forall \beta \in \mu,\{\beta \geqq \varepsilon\} \Rightarrow\left\{X^{\varepsilon}=X^{\beta}\right\}\right\}\right\}$ in which case the limit of the sequence is $X^{\varepsilon}$. We denote by $\operatorname{luis}(F)(D)$ the limit of a stationary upper iteration sequence for $F$ starting with $D$ (dually $l l i s(F)(D)$ ).

In the following the class of ordinals, the ordinal addition, the ordinal multiplication and the first infinite limit ordinal are respectively denoted by $\mathrm{Ord},+, \cdot$ and $\omega$ (the definition of + and $\cdot$ shall be used in the form stated by Birkhoff [3]).

The set of prefixed points of $F$ is prefp $(F)=\{X \in L: X \subseteq F(X)\}$. Dually postfp $(F)=\{X \in L: F(X) \subseteq X\}$. Therefore $f p(F)=\operatorname{prefp}(F) \cap$ postfp $(F)$.

We use Church [4]'s lambda notation (so that $F$ is $\lambda X . F(X)$ ). 


\section{Behavior of an upper iteration sequence.}

Lemma 3.1. Let $\left\langle X^{\delta}, \delta \in O r d\right\rangle$ be the Ord-termed upper iteration sequence for the monotone operator $F$ on the complete lattice $L(\subseteq, \perp, T, \cup, \cap)$ into itself starting with $D \in L$,

(1) $\forall P \in L,\{\{D \subseteq P\}$ and $\{F(P) \subseteq P\}\} \Rightarrow\left\{\forall \delta \in O r d, X^{\delta} \subseteq P\right\}$,

(2) $\{D \in \operatorname{postfp}(F)\} \Rightarrow\left\{\forall \delta \in O r d, X^{\delta} \subseteq D\right\}$.

Proof. Let $P \in L$ be such that $D \subseteq P$ and $F(P) \subseteq P$, then by Definition 2.1(a) $D=X^{0} \cong P$. Assume that $\forall \alpha \in \operatorname{Ord}, \quad\{\alpha<\delta\} \Rightarrow$ $\left\{X^{\alpha} \cong P\right\}$. If $\delta$ is a successor ordinal, then we have $X^{\delta-1} \subseteq P$ so that by monotony $F\left(X^{\delta-1}\right) \subseteq F(P) \leqq P$ proving by Definition 2.1(b) that $X^{\delta} \subseteq P$. If $\delta$ is a limit ordinal then by induction hypothesis and definition of least upper bounds $\mathrm{U}_{\alpha<\delta} X^{\alpha} \subseteq P$ proving by Definition 2.1(c) that $X^{\delta} \leqq P$. By transfinite induction $\forall \delta \in O r d, X^{\delta} \leqq P$. In particular when $D \in$ postfp $(F)$ we have $D \subseteq D$ and $F(D) \subseteq D$ which imply $\forall \delta \in O r d, X^{\delta} \subseteq D$.

THEOREM 3.2. Let $\left\langle X^{\delta}, \delta \in O r d\right\rangle$ be the Ord-termed upper iteration sequence for the monotone operator $F$ on the complete lattice $L(\subseteq, \perp, T, \cup, \cap)$ into itself starting with $D \in L$,

(1) $\forall \delta \in$ Ord, let $\beta \leqq \delta$ and $n<\omega$ be respectively the quotient and remainder of the ordinal division of $\delta$ by $\omega$ (i.e., $\delta=\beta \cdot \omega+n$ ), $\forall \beta^{\prime}: \beta^{\prime}>\beta, \forall \gamma: \beta^{\prime} \cdot \omega \leqq \gamma \leqq \beta^{\prime} \cdot \omega+n, X^{o} \leqq X^{\gamma}$.

(2) The subsequence $\left\langle X^{\alpha \cdot \omega}, \alpha \in \mu\right\rangle$ is a stationary increasing chain, its limit $X^{\eta \cdot \omega}$ is the least postfixed point of $F$ greater than or equal to $D$.

(3) There exists a smallest limit ordinal $\xi$ such that $\xi \leqq \eta \cdot \omega$ and $X^{\xi} \in \operatorname{prefp}(F) \underline{\cup}$ postfp $(F)$.

(4) If $X^{\xi} \in \operatorname{prefp}(F)$ then the subsequence $\left\langle X^{\delta}, \xi \leqq \delta<\xi+\mu\right\rangle$ (as well as $\left\langle X^{o}, \xi \leqq \delta\right\rangle$ ) is a stationary increasing chain of elements of prefp $(F)$, its limit luis $(F)\left(X^{\xi}\right)$ is equal to $X^{\eta \cdot \omega}$ which is the least of the fixed points of $F$ greater than or equal to $D$.

(5) If $X^{\xi} \in$ postfp $(F)$ then $\left\langle X^{\xi+n}, n \in \omega\right\rangle$ is a decreasing chain of elements of postfp $(F)$ and $\forall \delta \in O r d, X^{\xi+\delta}=X^{\xi+m}$ where $m$ is the remainder of the ordinal division of $\delta$ by $w$.

Proof.

(1) $\forall \delta \in O r d$, there exist unique $\beta$ and $n$ such that $\delta=\beta \cdot \omega+n$ and $\beta \leqq \delta, n<\omega$. If $\delta$ is a limit ordinal then $n=0$ and $\forall \beta^{\prime}>\beta$, $\beta^{\prime} \cdot \omega>\beta \cdot \omega=\delta$ and $\beta^{\prime} \cdot \omega$ is an infinite limit ordinal so that by Definition 2.1(c) $X^{\delta} \subseteq \bigcup_{\alpha<\beta^{\prime} \cdot \omega} X^{\alpha}=X^{\beta^{\prime} \cdot \omega}$. If $n \neq 0$ then $\delta$ is a successor ordinal and $(\delta-1)=\beta \cdot \omega+(n-1)$. Assume that $\forall \beta^{\prime}$ such that $\beta^{\prime}>\beta$ and $\forall \gamma$ such that $\beta^{\prime} \cdot \omega \leqq \gamma \leqq \beta^{\prime} \cdot \omega+(n-1)$ we have 
$X^{\delta-1} \cong X^{r}$. According to Definition 2.1(b) and by monotony $X^{\delta}=$ $F\left(X^{\delta-1}\right) \cong F\left(X^{r}\right)=X^{\gamma+1}$. Also $X^{\delta} \cong X^{\beta^{\prime} \omega}$ therefore with $\gamma^{\prime}=\gamma+1$ we get $\forall \beta^{\prime}: \beta^{\prime}>\beta, \forall \gamma^{\prime}: \beta^{\prime} \cdot \omega \leqq \gamma^{\prime} \leqq \beta^{\prime} \cdot \omega+n, X^{\delta} \leqq X^{\gamma^{\prime}}$. By transfinite induction on $\delta$ Theorem 3.2(1) is proved.

(2) By 3.2(1) the subsequence $\left\langle X^{\alpha \cdot \omega}, \alpha \in \mu\right\rangle$ is an increasing chain. Assume that $\left\{\forall \eta \in O r d,\{\eta \in \mu\right.$ and $(\eta+1) \in \mu\} \Rightarrow\left\{X^{\eta \cdot \omega} \neq\right.$ $\left.X^{(\eta+1) \cdot \omega}\right\}$. This implies that $\left\langle X^{\alpha \cdot \omega}, \alpha \in \mu\right\rangle$ is a strictly increasing chain so that the class $\left\{X^{\alpha \cdot \omega}: \alpha \in \mu\right\}$ is equipotent with the class $\{\alpha \cdot \omega: \alpha \in \mu\}$. Since $\lambda \alpha .(\alpha \cdot \omega)$ is a one-one function mapping $\{\alpha: \alpha \in \mu\}$ onto $\{\alpha \cdot \omega: \alpha \in \mu\}$ the class $\left\{X^{\alpha \cdot \omega}: \alpha \in \mu\right\}$ is equipotent with the class $\{\alpha: \alpha \in \mu\}$. Therefore by definition of $\mu$ we have Card $\left(\left\{X^{\alpha \cdot \omega}: \alpha \in \mu\right\}\right)>$ Card $(L)$ and also by $\left\{\forall \alpha \in \mu, X^{\alpha \cdot \omega} \in L\right\}$ we obtain the contradiction Card $\left(\left\{X^{\alpha \cdot \omega}: \alpha \in \mu\right\}\right) \leqq \operatorname{Card}(L)$. By reductio ad absurdum $\{\exists \eta:(\eta \in \mu)$ and $((\eta+1) \in \mu)$ and $\left.X^{\eta \cdot \omega}=X^{(\eta+1) \cdot \omega}\right\}$.

Since $(\eta \cdot \omega)+1<(\eta+1) \cdot \omega$ and $(\eta+1) \cdot \omega$ is an infinite limit ordinal Definitions 2.1(b) and 2.1(c) imply that $F\left(X^{\eta \cdot \omega}\right)=X^{(\eta \cdot \omega)+1} \subseteq$ $\mathrm{U}_{\alpha<(\eta+1) \cdot \omega} X^{\alpha}=X^{(\eta+1) \cdot \omega}=X^{\eta \cdot \omega}$. Also $D=X^{0} \subseteq \bigcup_{\alpha<\eta \cdot \omega} X^{\alpha}=X^{\eta \cdot \omega}$ so that $X^{\eta \cdot \omega}$ is a postfixed point of $F$ greater than or equal to $D$. Let $P \in L$ be such that $F(P) \subseteq P$ and $D \subseteq P$. Then Lemma 3.1(1) implies that $X^{\eta \cdot \omega} \subseteq P$ proving that $X^{\eta \cdot \omega}$ is the least postfixed point of $F$ greater than or equal to $D$.

$\forall \alpha \in \operatorname{Ord}, \alpha>\eta$ implies $\alpha \cdot \omega>\eta \cdot \omega$ and therefore by Definition 2.1(c) $X^{\alpha \cdot \omega}=\bigcup_{\beta<\alpha \cdot \omega} X^{\beta}=X^{\eta \cdot \omega} \cup\left(\bigcup_{\eta \cdot \omega \leqq \beta<\alpha \cdot \omega} X^{\beta}\right)$. But $X^{\eta \cdot \omega} \in \operatorname{postfp}(F)$ so that according to Lemma $3.1(2), \forall \beta \geqq \eta \cdot \omega$ we have $X^{\beta} \cong X^{\eta \cdot \omega}$ proving that $X^{\alpha \cdot \omega}=X^{\eta \cdot \omega}$ and that $\left\langle X^{\alpha \cdot \omega}, \alpha \in \mu\right\rangle$ and $\left\langle X^{\alpha \cdot \omega}, \alpha \in\right.$ Ord $\rangle$ are stationary.

(The following Theorem 4.1 will show that $X^{\eta \cdot \omega}$ can be constructed more directly as luis $(\lambda X . X \cup F(X))(D)=$ luis $(\lambda X . D \cup F(X))(D))$.

(3) Since $X^{\eta \cdot \omega} \in$ postfp $(F)$ and Ord is well-ordered there exists a smallest limit ordinal $\xi \leqq \eta \cdot \omega$ such that $X^{\xi}$ and $F\left(X^{\xi}\right)$ are comparable.

(4) If $X^{\xi} \in \operatorname{prefp}(F)$ then by monotony of $F$, Definition 2.1 and transfinite induction, it is easy to prove that $\{\forall \delta, \beta \in \operatorname{Ord}$, $\left.\{\xi \leqq \delta \leqq \beta\} \Rightarrow\left\{D \subseteq X^{\delta} \subseteq X^{\beta} \cong F\left(X^{\beta}\right)\right\}\right\}$. By definition of $\mu$ the increasing subchain $\left\langle X^{\delta}, \xi \leqq \delta\langle\xi+\mu\rangle\right.$ of elements of $L$ cannot be strictly increasing so that $\left\{\exists \varepsilon \in \operatorname{Ord}:(\xi \leqq \varepsilon<\varepsilon+1<\xi+\mu)\right.$ and $\left.\left(X^{\varepsilon}=X^{\varepsilon+1}\right)\right\}$. Then by transfinite induction using Definition 2.1 it is immediate that $\left\langle X^{\delta}, \xi \leqq \delta\langle\xi+\mu\rangle\right.$ and $\left\langle X^{\delta}, \xi \leqq \delta\right\rangle$ are stationary of limit $X^{\varsigma}$. Since $D \cong X^{\varepsilon}=X^{\varepsilon+1}=F\left(X^{\varepsilon}\right), X^{\varepsilon}$ is a fixed (and postfixed) point of $F$ greater than or equal to $D$. Let $P \in L$ be such that $D \cong P$ and $F(P) \subseteq P$. By Lemma 3.1(1) we have $X^{\varepsilon} \cong P$ proving that $X^{\varepsilon}$ is the least fixed (and postfixed) point of $F$ greater than or equal to $D$. Moreover $X^{\varepsilon}=X^{\eta \cdot \omega}$ by $3.2(2)$.

(5) When $F\left(X^{\xi}\right) \subseteq X^{\xi}$ it is easy, using the monotony of $F$, to 
prove by finite induction that the subsequence $\left\langle X^{\xi+n}, n \in \omega\right\rangle$ is a decreasing chain. If $\delta=0$ then $\delta=0 \cdot \omega+0$ and obviously $X^{\xi+\delta}=$ $X^{\xi}=X^{\xi+0}$. Assume that $\forall \alpha \in \operatorname{Or} d,\{\alpha<\delta\} \Rightarrow\left\{X^{\xi+\alpha}=X^{\xi+m}\right\}$ where $m$ is the remainder of the ordinal division of $\alpha$ by $\omega$. If $\delta$ is a successor ordinal then $\exists \beta \in \operatorname{Ord}, \exists n \in \omega$ such that $\delta=\beta \cdot \omega+n$ with $n \neq 0$. Hence $\delta-1=\beta \cdot \omega+(n-1)$ so that by induction hypothesis $X^{(\xi+\delta)-1}=X^{\xi+(\delta-1)}=X^{\xi+(n-1)}=X^{(\xi+n)-1}$. By Definition 2.1(b), $X^{\xi+\delta}=$ $F\left(X^{(\xi+\delta)-1}\right)=F\left(X^{(\xi+n)-1}\right)=X^{\xi+n}$. If $\delta$ is a limit ordinal then $\xi+\delta$ is a limit ordinal because $\xi$ is a limit ordinal. Hence by Definition 2.1(c) $X^{\xi+\delta}=\mathbf{U}_{r<\xi+\delta} X^{r}=\left(\mathbf{U}_{r<\xi} X^{r}\right) \cup\left(\mathbf{U}_{\xi \leq r<\xi+\delta} X^{r}\right)=X^{\xi} \cup\left(\mathbf{U}_{r<\delta} X^{\xi+r}\right)=X^{\xi}$ since $X^{\xi} \in$ postfp $(F)$ implies according to Lemma 3.1(2) that $\forall \gamma, X^{\xi+r} \subseteq X^{\xi}$. By transfinite induction, $\forall \delta \in O r d, X^{\xi+\delta}=X^{\xi+n}$ where $n$ is the remainder of the ordinal division of $\delta$ by $\omega$.

The following corollary is immediate from 3.2(4):

COROLLARY 3.3. (Behavior of an upper iteration sequence starting from a prefixed point of $F$.) $A$ e-termed upper iteration sequence $\left\langle X^{\delta}, \delta \in \mu\right\rangle$ for $F$ starting with $D \in$ prefp $(F)$ is a stationary increasing chain, its limit luis $(F)(D)$ is the least of the fixed points of $F$ greater than or equal to $D$.

An upper closure operator $\bar{\rho}$ on $L$ into $L$ is monotone, extensive $(\forall X \in L, X \subseteq \bar{\rho}(X))$ and idempotent $(\forall X \in L, \bar{\rho}(\bar{\rho}(X))=\bar{\rho}(X))$. Dually, a lower closure operator $\underline{\rho}$ on $L$ into $L$ is monotone, reductive $(\forall X \in L, \underline{\rho}(X) \subseteq X)$ and idempotent.

CoRollary 3.4. The restriction of luis $(F)$ to prefp $(F)$ is an upper closure operator.

Proof. $\forall D \in$ prefp $(F)$, we have luis $(F)(D) \in f p(P) \leqq$ prefp $(F)$. By 3.3, $D \subseteq l u i s(F)(D)$. By transfinite induction it is easy to show that the upper iteration sequence $\left\langle X^{\delta}, \delta \in \mu\right\rangle$ for $F$ starting with a fixed point $P$ of $F$ is such that $\left\{\forall \delta \in \mu, P=X^{\delta}\right\}$ so that in particular for $P=\operatorname{luis}(F)(D)$ we have $\operatorname{luis}(F)(\operatorname{luis}(F)(D))=\operatorname{luis}(F)(D)$. Finally by transfinite induction it is easy to show that the upper iteration sequences $\left\langle X^{\delta}, \delta \in \mu\right\rangle$ and $\left\langle Y^{\delta}, \delta \in \mu\right\rangle$ starting respectively by prefixed points $D$ and $E$ of $L$ satisfying $D \subseteq E$ are such that $\left\{\forall \delta \in \mu, X^{\delta} \subseteq Y^{\delta}\right\}$. Therefore by Theorem 3.3, $\exists \varepsilon \in \mu, \exists \varepsilon^{\prime} \in \mu$ such that luis $(F)(D)=X^{\varepsilon}=X^{\max \left(\varepsilon, \varepsilon^{\prime}\right)} \subseteq Y^{\max \left(\varepsilon, \varepsilon^{\prime}\right)}=Y^{\varepsilon^{\prime}}=$ luis $(F)(E)$.

Applying the duality principle, we get:

COROLLARY 3.5. The restriction of llis $(F)$ to postfp $(F)$ is a 
lower closure operator.

4. Constructive characterization of the sets of pre- and postfixed points of $F$.

THEOREM 4.1. The $\mu$-termed upper iteration sequences $\left\langle X^{\delta}, \delta \in \mu\right\rangle$ and $\left\langle Y^{\delta}, \delta \in \mu\right\rangle$ for $\lambda X \cdot X \cup F(X)$ and $\lambda X \cdot D \cup F(X)$ respectively, starting with an arbitrary element $D$ of the complete lattice $L$ are stationary increasing chains such that $\forall \delta \in \mu, X^{\delta}=Y^{\delta}$. Their limits luis $(\lambda X \cdot X \cup F(X))(D)$ and luis $(\lambda X \cdot D \cup F(X))(D)$ are equal to the least of the postfixed points of $F$ greater than or equal to $D$.

Proof. 4.1.1. $\forall D \in L, D$ is a prefixed point of $\lambda X \cdot X \cup F(X)$ and $\lambda X \cdot D \cup F(X)$ which are monotone operators on the complete lattice $L$ into itself. Hence Theorem 3.3 implies that $\left\langle X^{o}, \delta \in \mu\right\rangle$ and $\left\langle Y^{\delta}, \delta \in \mu\right\rangle$ are stationary increasing chains.

\subsection{2. $\forall \delta \in \mu, X^{\delta}=Y^{\delta}$.}

By Definitions 2.1(a) and 2.1(b) the lemma is true for $\delta=0$ and $\delta=1$. Assume it is true for every $\gamma$ such that $2 \leqq \gamma<\delta<\mu$. If $\delta$ is the successor of a successor ordinal then $X^{\delta}=X^{\delta-1} \cup F\left(X^{\delta-1}\right)=$ $Y^{\delta-1} \cup F\left(Y^{\delta-1}\right)=D \cup F\left(Y^{\delta-2}\right) \cup F\left(Y^{\delta-1}\right)=D \cup F\left(Y^{\delta-1}\right)=Y^{\delta}$ by Definition 2.1(b), induction hypothesis, 4.1.1 and monotony of $F$. If $\delta$ is the successor of a limit ordinal then Definition 2.1(b), induction hypothesis, 4.1.1, Definition 2.1(c) and definition of least upper bounds imply $X^{\delta}=X^{\delta-1} \cup F\left(X^{\delta-1}\right)=Y^{\delta-1} \cup F\left(Y^{\delta-1}\right)=\left(\bigcup_{\alpha<\delta-1} Y^{\alpha}\right) \cup F\left(Y^{\delta-1}\right)=$ $\bigcup_{\alpha<\delta-1}\left(Y^{\alpha+1} \cup F\left(Y^{\delta-1}\right)\right)=\bigcup_{\alpha<\delta-1}\left(D \cup F\left(Y^{\alpha}\right) \cup F\left(Y^{\delta-1}\right)\right)=D \cup F\left(Y^{\delta-1}\right)=$ $Y^{\delta}$. If $\delta$ is a limit ordinal then Definition 2.1(c) and induction hypothesis imply $X^{\delta}=\bigcup_{\alpha<\delta} X^{\alpha}=\bigcup_{\alpha<\delta} Y^{\alpha}=Y^{\delta}$. By transfinite induction the lemma is true for every $\delta \in \mu$.

4.1.3. By 4.1.1 and 4.1.2 the limits luis $(\lambda X \cdot X \cup F(X))(D)$ and luis $(\lambda X \cdot D \cup F(X))(D)$ exist and are equal. By 3.3 luis $(\lambda X \cdot X \cup$ $F(X))(D)$ is the least of the fixed points of $\lambda X \cdot X \cup F(X)$ greater than or equal to $D$ so that $\{\forall P \in L,\{P=P \cup F(P)\} \Leftrightarrow\{F(P) \subseteq P\}\}$ implies that luis $(\lambda X \cdot X \cup F(X))(D)$ and luis $(\lambda X \cdot D \cup F(X))(D)$ are equal to the least of the postfixed points of $F$ greater than or equal to $D$.

COROLLARY 4.2. The set of postfixed points of $F$ is a nonempty complete lattice:

$$
\text { postfp }(F)(\subseteq, l f p(F), T, \lambda S \cdot l u i s(\lambda Z \cdot Z \cup F(Z))(\cup S), \cap)
$$

where the least fixed point of $F$ is lfp $(F)=$ luis $(F)(D)=\cap\{X \in L$ : $F(X) \subseteq X\}$ for every $D \in L$ such that $D \subseteq l f p(F)$. 
Proof. By 4.1 and 3.4 the image of the nonempty complete lattice $L$ by the upper closure operator $\bar{\rho}=\operatorname{luis}(\lambda Z \cdot Z \cup F(Z))$ is included in postfp $(F)$. Reciprocally, $\forall P \in \operatorname{postfp}(F)$ we know that $P \in f p(\lambda Z \cdot Z \cup F(Z))$ so that the upper iteration sequence $\left\langle X^{\delta}, \delta \in \mu\right\rangle$ for $\lambda Z \cdot Z \cup F(Z)$ starting with $P$ is such that $\left\{\forall \delta \in \mu, P=X^{\circ}\right\}$. Hence $\bar{\rho}(P)=P$ that is postfp $(F) \leqq \bar{\rho}(L)$ and by antisymmetry we have postfp $(F)=\bar{\rho}(L)$.

By Ward [21]'s theorem $\bar{\rho}(L)$ is a nonempty complete lattice $(\subseteq, \bar{\rho}(\perp), T, \lambda S \cdot \bar{\rho}(\cup S), \cap)$.

Also by 4.1 luis $(\lambda Z \cdot Z \cup F(Z))(\perp)=\operatorname{luis}(\lambda Z \cdot \perp \cup F(Z))(\perp)=$ luis $(F)(\perp)=\cap$ postfp $(F)$ by definition of the infimum of a complete lattice. By 3.3 luis $(F)(\perp)$ is the least of the fixed points of $F$ greater than or equal to $\perp$, therefore it is the least fixed point of $F$.

Finally let $D \in L$, be such that $D \subseteq l f p(F)$ and $\left\langle X^{o}, \delta \in \mu\right\rangle$, $\left\langle Y^{\delta}, \delta \in \mu\right\rangle,\left\langle Z^{\delta}, \delta \in \mu\right\rangle$ be the upper iteration sequences for $F$ respectively starting with $\perp, D$, and $l f p(F)$. By transfinite induction it is immediate that $\left\{\forall \delta \in \mu, X^{\delta} \subseteq Y^{\delta} \subseteq Z^{\delta}=l f p(F)\right\}$. According to 3.3, $\left\langle X^{\delta}, \delta \in \mu\right\rangle$ is stationary and its limit luis $(F)(\perp)$ is $\operatorname{lfp}(F)$. Therefore $\left\langle Y^{\delta}, \delta \in \mu\right\rangle$ is stationary of limit lfp $(F)$.

Applying the duality principle, we obtain:

CoROLlaRy 4.3. The set of prefixed points of $F$ is a nonempty complete lattice:

$$
\operatorname{prefp}(F)(\subseteq, \perp, g f p(F), \cup, \lambda S \cdot \operatorname{lis}(\lambda Z \cdot Z \cap F(Z))(\cap S))
$$

where the greatest fixed point of $F$ is gfp $(F)=l l i s(F)(D)=\cup\{X \in L$ : $X \subseteq F(X)\}$ for every $D \in L$ such that $g f p(F) \subseteq D$.

Let $\left\{F_{i}: i \in I\right\}$ be a family of monotone maps from $L$ into $L$. The unary polynomials of the algebra $\left\langle L ; U, \cap,\left\{F_{i}: i \in I\right\}\right\rangle$ are mappings on $L$ into $L$ defined as follows:

(i) The identity mapping $\lambda X \cdot X$ is a unary polynomial.

(ii) For every $i \in I$, if $P$ is an unary polynomial then so is $\lambda X \cdot F_{i}(P(X))$.

(iii) If $\left\{P_{\gamma}: \gamma \in J\right\}$ is a family of unary polynomials then so are $\lambda X \cdot \mathrm{U}_{r \in J} P_{\gamma}(X)$ and $\lambda X \cdot \bigcap_{r \in J} P_{r}(X)$.

(iv) Unary polynomials are those and only those which we get from (i), (ii), and (iii).

Since polynomials are functions of $L$ into $L$ they are ordered by the pointwise ordering $\{F \subseteq G\} \Leftrightarrow\{\forall X \in L, F(X) \subseteq G(X)\}$.

COROLLARY 4.4. Every unary polynomial of $\left\langle L ; \cup, \cap,\left\{F_{i}: i \in I\right\}\right\rangle$ 
is less than or equal to $\lambda X \cdot \operatorname{luis}\left(\lambda Z \cdot Z \cup\left(\bigcup_{i \in I} F_{i}(Z)\right)\right)(X)$ and greater than or equal to $\lambda X \cdot \operatorname{llis}\left(\lambda Z \cdot Z \cap\left(\bigcap_{i \in I} F_{i}(Z)\right)\right)(X)$.

Proof. Let $\bar{F}$ be $\lambda Z \cdot\left(Z \cup\left(\bigcup_{i \in I} F_{i}(Z)\right)\right)$ and $\underline{F}$ be $\lambda Z \cdot(Z \cap$ $\left.\left(\bigcap_{i \in I} F_{i}(Z)\right)\right), \bar{F}$ and $\underline{F}$ are monotone maps on $L$ into $L$. The proof is by induction on the structure of unary polynomials:

(i) luis $(\bar{F})$ is extensive and $l l i s(E)$ is reductive so that for every $X$ of $L$ we have llis $(\underline{F})(X) \subseteq X \subseteq l u i s(\bar{F})(X)$.

(ii) Let $P$ be a unary polynomial such that for every $X$ of $L$ we have llis $(\underline{F})(X) \cong P(X) \subseteq l u i s(\bar{F})(X)$. Then for every monotone $F_{i}$, we have $F_{i}($ llis $(\underline{F})(X)) \subseteq F_{i}(P(X)) \subseteq F_{i}(l u i s(\bar{F})(X))$. But $\operatorname{llis}(\underline{F})(X)=\underline{F}(l l i s(\underline{F})(X)) \leqq F_{i}(l l i s(\underline{F})(X))$ and dually $F_{i}(l u i s(\bar{F})(X)) \leqq$ $\bar{F}($ luis $(\bar{F})(X))=$ luis $(\bar{F})(X)$ so that by transitivity llis $(\underline{F})(X) \leqq$ $F_{i}(P(X)) \subseteq \operatorname{luis}(\bar{F})(X)$.

(iii) Let $\left\{P_{\gamma}: \gamma \in J\right\}$ be a family of unary polynomials such that for every $X \in L$, llis $(\underline{F})(X) \subseteq P_{\gamma}(X) \subseteq l u i s(\bar{F})(X)$ then by definition of least upper bounds we have llis $(\underline{F})(X) \subseteq \bigcup_{r \in J} P_{r}(X) \subseteq l u i s(\bar{F})(X)$ and by definition of greatest lower bounds we have llis $(\underline{F})(X) \subseteq$ $\bigcap_{r \in J} P_{r}(X) \subseteq \operatorname{luis}(\bar{F})(X)$.

The generalization of 4.4 to $n$-ary polynomials is immediate.

5. Constructive characterization of the set of fixed points of $F$.

THEOREM 5.1. (Constructive version of Tarski's lattice theoretical fixed point theorem.) The set of fixed points of $F$ is a nonempty complete lattice with ordering $\subseteq$, infimum luis $(F)(\perp)$, supremum llis $(F)(T)$, least upper bound $\lambda S \cdot$ luis $(F)(\cup S)$ and greatest lower bound $\lambda S \cdot \operatorname{llis}(F)(\cap S)$.

Proof. By Theorems 3.3 and 3.4, $f p(F)$ is the image of $\operatorname{prefp}(F)$ by the upper closure operator luis $(F)$ and by Theorem $4.3 \operatorname{prefp}(F)$ is a nonempty complete lattice so that by Ward [21]'s theorem $f p(F)$ is a nonempty complete lattice with ordering $\subseteq$, infimum luis $(F)(\perp)$ and least upper bound $\lambda S \cdot l u i s(F)(\cup S)$. By duality, $f p(F)$ is the image of the nonempty complete lattice postfp $(F)$ by the lower closure operator llis $(F)$ so that the supremum of $F$ is llis $(F)(T)$ and the greatest lower bound $\lambda S \cdot l l i s(F)(\cap S)$.

The construction of extremal fixed points of monotone operators as limits of stationary transfinite iteration sequences may be found in Devidé [7] (where lfp $(\lambda Z \cdot D \cup F(Z)$ ) is the limit of the sequence $X^{0}=D, X^{\delta}=X^{\delta-1} \cup F\left(X^{\delta-1}\right)$ for successor ordinals and $X^{\delta}=\mathrm{U}_{\alpha<\delta} X^{\alpha}$ 
for limit ordinals) in Hitchcock and Park [8] (where $l f p(F)$ is the limit of $X^{0}=\perp, X^{\delta}=\bigcup_{\alpha<\delta} F\left(X^{\alpha}\right)$ for every nonzero ordinal) and in Pasini [15] (where transfinite sequences are defined as in Definition 2.1).

CoRollary 5.2. Let $D$ be an arbitrary element of $L$. luis $(F) \circ$ llis $(\lambda Z \cdot Z \cap F(Z))(D)$ and llis $(F) \circ$ luis $(\lambda Z \cdot Z \cup F(Z))(D)$ are fixed points of $F$ greater than or equal to any fixed point of $F$ less than or equal to $D$ and less than or equal to any fixed point of $F$ greater than or equal to $D$. Moreover luis $(F) \circ l l i s(\lambda Z \cdot Z \cap F(Z))(D) \subseteq$ llis $(F) \circ$ luis $(\lambda Z \cdot Z \cup F(Z))(D)$.

Proof. Assume that $A$ is a fixed point of $F$ less than or equal to $D$ and $B$ a fixed point of $F$ greater than or equal to $D$, that is $F(A)=A \subseteq D \subseteq B=F(B)$. Then by monotony (3.4, 3.5) and fixed point property $A=$ luis $(F) \circ$ llis $(\lambda Z \circ Z \cap F(Z))(A) \subseteq l u i s(F) \circ$ llis $(\lambda Z \cdot Z \cap F(Z))(D) \leqq$ luis $(F) \circ \operatorname{llis}(\lambda Z \cdot Z \cap F(Z))(B)=B$. The same way, $A \subseteq l$ lis $(F) \circ$ luis $(\lambda Z \cdot Z \cup F(Z))(D) \subseteq B$.

Let $P$ be llis $(\lambda Z \cdot Z \cap F(Z))(D)$ and $Q$ be luis $(\lambda Z \cdot Z \cup F(Z))(D)$. Let $S$ be $\{X \in L: P \subseteq X \subseteq Q\} . \quad S$ is a complete sublattice of $L$ with infimum $P$ and supremum $Q$. By 4.1 and its dual $P \subseteq F(P)$ and $F(Q) \subseteq Q$ so that $F(S) \subseteq S$. Then by 5.1 the least fixed point of $F$ restricted to $S$ is luis $(F)(P)$ and the greatest fixed point of $F$ restricted to $S$ is llis $(F)(Q)$ proving that luis $(F) \circ l l i s(\lambda Z \cdot Z \cap$ $F(Z))(D) \subseteq l$ lis $(F) \circ$ luis $(\lambda Z \cdot Z \cup F(Z))(D)$.

A lower preclosure operator $\rho$ on $L$ is monotone, idempotent and satisfies the lower connectivity axiom $\{\forall X \in L, \underline{\rho}(X \cap \underline{\rho}(X))=\underline{\rho}(X)\}$. An upper preclosure operator $\bar{\rho}$ on $L$ is monotone, idempotent and satisfies the upper connectivity axiom $\{\forall X \in L, \bar{\rho}(X \cup \bar{\rho}(X))=\bar{\rho}(X)\}$.

CoRollary 5.3. The set $f p(F)$ of fixed points of $F$ is the image of $L$ by the lower preclosure operator luis $(F) \circ$ llis $(\lambda Z \cdot Z \cap F(Z))$ and the image of $L$ by the upper preclosure operator llis $(F) \circ$ luis $(\lambda Z \cdot Z \cup F(Z))$.

Proof. luis $(F) \circ$ llis $(\lambda Z \cdot Z \cap F(Z))$ is a lower preclosure operator since it is the composition of the upper closure operator luis $(F)$ and the lower closure operator $\operatorname{llis}(\lambda Z \cdot Z \cap F(Z))(3.4,4.1$ and 3.5, Ladegaillerie [12]). By duality $\operatorname{llis}(F) \circ \operatorname{luis}(\lambda Z \cdot Z \cup F(Z))$ is an upper preclosure operator.

Cousot and Cousot [5] already used the idea of constructing (or approximating) the fixed points of monotone operators by means of 
an upper iteration sequence followed by a lower iteration sequence. This idea was also used by Manna and Shamir [13] and our results $3.3,4.1,5.2$, and 5.3 improve their results obtained on the more restricted model of continuous functional equations on functions of flat lower semi-lattices.

6. Constructive characterization of the set of fixed points of a family of commuting operators.

LEMMA 6.1. Let $\underline{F}$ and $\bar{F}$ be monotone operators on the nonempty complete lattice $L(\subseteq, \perp, T, \cup, \cap)$ into itself such that $\bar{F} \circ \underline{F} \cong$ $\underline{F} \circ \bar{F}$ and $\underline{F} \subseteq \bar{F}$ (i.e., $\forall X \in L, \bar{F}(\underline{F}(X)) \subseteq \underline{F}(\bar{F}(X))$ and $\underline{F}(X) \subseteq \bar{F}(X))$. The set of common fixed points of $\bar{F}$ and $\underline{F}$ is a nonempty complete lattice:

$f p(\underline{F}, \bar{F})(\subseteq, l f p(\bar{F}), g f p(\underline{F}), \lambda S \cdot l u i s(\bar{F})(\cup S), \lambda S \cdot l l i s(\underline{F})(\cap S))$

which is the image of $L$ by luis $(\bar{F}) \circ$ llis $(\lambda Z \cdot Z \cap \underline{F}(Z))$ and the image of $L$ by llis $(\underline{F}) \circ l u i s(\lambda Z \cdot Z \cup \bar{F}(Z))$.

Proof.

6.1.1. $\forall D \in \operatorname{prefp}(\underline{F}), \underline{F}($ luis $(\bar{F})(D))=$ luis $(\bar{F})(D)$.

Since $D \in \operatorname{prefp}(\underline{F})$ and $\underline{F} \subseteq \bar{F}$ we have $D \subseteq \underline{F}(D) \subseteq \bar{F}(D)$ so that the upper iteration sequence $\left\langle X^{\delta}, \delta \in \mu\right\rangle$ for $\bar{F}$ starting with $D$ is stationary, its limit luis $(\bar{F})(D)$ is a fixed point of $\bar{F}$ (3.3). Again since $\underline{F} \subseteq \bar{F}$ we have $\underline{F}($ luis $(\bar{F})(D)) \subseteq \bar{F}($ luis $(\bar{F})(D))=$ luis $(\bar{F})(D)$.

Let us show that $\left\{\forall \delta \in \mu, X^{o} \subseteq \underline{F}\left(X^{o}\right)\right\}$. For $\delta=0$ we have $X^{0}=$ $D \subseteq \underline{F}\left(X^{0}\right)$ since $D \in \operatorname{prefp}(\underline{F})$. Assume that the lemma is true for all $\alpha<\delta<\mu$. If $\delta$ is a successor ordinal then in particular $X^{\delta-1} \subseteq$ $\underline{F}\left(X^{\delta-1}\right)$. Since $\bar{F}$ is monotone and $\bar{F} \circ \underline{F} \subseteq \boldsymbol{F} \circ \bar{F}$ we have by Definition 2.1(b), $X^{\delta}=\bar{F}\left(X^{\delta-1}\right) \leqq \bar{F}\left(\underline{F}\left(X^{\delta-1}\right)\right) \leqq \underline{F}\left(\bar{F}\left(X^{\delta-1}\right)\right)=\underline{F}\left(X^{o}\right)$. If $\delta$ is a limit ordinal then $X^{\alpha} \subseteq F\left(X^{\alpha}\right)$ for every $\alpha<\delta$. By 2.1(c) and monotony, $X^{\delta}=\bigcup_{\alpha<\delta} X^{\alpha} \subseteq \bigcup_{\alpha<\delta} \underline{F}\left(X^{\alpha}\right) \subseteq \underline{F}\left(\bigcup_{\alpha<\delta} X^{\alpha}\right)=\underline{F}\left(X^{\delta}\right)$. By transfinite induction the lemma is true for every $\delta \in \mu$.

By 3.3, luis $(\bar{F})(D)$ is the limit of $\left\langle X^{\delta}, \delta \in \mu\right\rangle$ so that luis $(\bar{F})(D) \subseteq$ $\underline{F}($ luis $(\bar{F})(D))$. By antisymmetry we conclude that luis $(\bar{F})(D)=$ $\underline{F}($ luis $(\bar{F})(D))$.

6.1.2. Let $D$ be an arbitrary element of $L$, then by the dual of Theorem 4.1, llis $(\lambda Z \cdot Z \cap \underline{F}(Z))(D) \in \operatorname{prefp}(F) \subseteq \operatorname{prefp}(\bar{F})$ so that Theorem 3.3 implies that luis $(\bar{F}) \circ l l i s(\lambda Z \cdot Z \cup \underline{F}(Z))(D) \in f p(\bar{F})$. Also by $\quad 6.1 .1$ luis $(\bar{F}) \circ$ llis $(\lambda Z \cdot Z \cap \underline{F}(Z))(D) \in f p(F)$. Consequently luis $(\bar{F}) \circ \operatorname{llis}(\lambda Z \cdot Z \cap F(Z))(L) \subseteq f p(\underline{F}) \cap f p(\bar{F})=f p(\underline{F}, \bar{F})$ and $f p(\underline{F}, \bar{F})$ is not empty (take $D$ equal to $\perp$ ).

Let $P \in f p(F, \bar{F})$ then $P \in L$ and luis $(\bar{F}) \circ l l i s(\lambda Z \cdot Z \cap F(Z))(P)$ is equal to $P$ since $\underline{F}(P)=P$ and $\bar{F}(P)=P$. Therefore $f p(\underline{F}, \bar{F}) \subseteq$ luis $(\bar{F}) \circ$ llis $(\lambda Z \cdot Z \cap \underline{F}(Z))(L)$ so that by antisymmetry we conclude 
$f p(\underline{F}, \bar{F})=l u i s(\bar{F}) \circ l l i s(\lambda Z \cdot Z \cap F(Z))(L)$.

6.1.3. By 4.3 llis $(\lambda Z \cdot Z \cap \underline{F}(Z))(L)$ is a nonempty complete lattice $\operatorname{prefp}(\underline{F})(\subseteq, \perp, g f p(\underline{F}), \cup, \lambda S \cdot l l i s(\lambda Z \cdot Z \cap \underline{F}(Z))(\cap S))$. By 3.4 luis $(\bar{F})$ is an upper closure operator so that by 6.1.2 and Ward [21]'s theorem $f p(\underline{F}, \bar{F})$ is a nonempty complete lattice with ordering $\subseteq$, infimum luis $(\bar{F})(\perp)=l f p(\bar{F})$ and least upper bound operation $\lambda S \cdot \operatorname{luis}(\bar{F})(\cup S)$.

The remaining parts of Lemma 6.1 are obtained by duality, $f p(\underline{F}, \bar{F})$ is the image of the nonempty complete lattice postfp $(\bar{F})$ $(\subseteq$, lfp $(\bar{F}), T, \lambda S \cdot l u i s(\lambda Z \cdot Z \cup \bar{F}(Z))(\cup S), \cap)$ by the lower closure operation llis $(\underline{F})$ so that the supremum of $f p(\underline{F}, \bar{F})$ is $l$ lis $(\underline{F})(T)=$ $g f p(\underline{F})$ and the greatest lower bound operation is $\lambda S \cdot l l i s(\underline{F})(\cap S)$.

THEOREM 6.2. (Constructive version of Tarski's generalized lattice theoretical fixed point theorem.) Let $\left\{F_{i}: i \in I\right\}$ be a nonempty family of monotone commuting operators on the nonempty complete lattice $L(\subseteq, \perp, T, \cup, \cap)$ into itself. The set of all common fixed points $f p\left(\left\{F_{i}: i \in I\right\}\right)$ of all the operators $\left\{F_{i}: i \in I\right\}$ is a nonempty complete lattice with ordering $\cong$, infimum lfp $\left(\lambda Z \cdot \bigcup_{i \in I} F_{i}(Z)\right)$, supremum $g f p\left(\lambda Z \cdot \bigcap_{i \in I} F_{i}(Z)\right)$, least upper bound operation $\lambda S \cdot$ luis $\left(\lambda Z \cdot \bigcup_{i \in I} F_{i}(Z)\right)(\cup S)$ and greatest lower bound operation $\lambda S \cdot \operatorname{llis}\left(\lambda Z \cdot \bigcap_{i \in I} F_{i}(Z)\right)(\cap S)$.

Proof.

6.2.1. Let $\bar{F}$ be $\lambda Z \cdot \bigcup_{i \in I} F_{i}(Z)$ and $\underline{F}$ be $\lambda Z \cdot \bigcap_{i \in I} F_{i}(Z)$. $\quad \bar{F}$ and $\underline{F}$ are monotone operators on $L$ into itself such that $\underline{F} \leqq \vec{F}$. $\forall X \in L, \forall i \in I$, we have $\bar{F}\left(F_{i}(X)\right)=\bigcup_{j \in I} F_{j}\left(F_{i}(X)\right)=\bigcup_{j \in I} F_{i}\left(F_{j}(X)\right) \subseteq$ $F_{i}\left(\bigcup_{j \in I} F_{j}(X)\right)=F_{i}(\bar{F}(X))$ by monotony and the commuting property. Therefore $\forall X \in L, \quad \bar{F}(\underline{F}(X))=\vec{F}\left(\bigcap_{i \in I} F_{i}(X)\right) \leqq \bigcap_{i \in I} \bar{F}\left(F_{i}(X)\right) \subseteq$ $\bigcap_{i \in I} F_{i}(\bar{F}(X))=\underline{F}(\bar{F}(X))$.

6.2.2. Clearly $f p\left(\left\{F_{i}: i \in I\right\}\right) \subseteq f p(\bar{F}, \underline{F})$ since $\left\{\forall i \in I, F_{i}(X)=X\right\}$ implies $\bar{F}(X)=\bigcup_{i \in I} F_{i}(X)=\bigcup_{i \in I} X=X$ and dually $\underline{F}(X)=X$. Whenever $X \in f p(\bar{F}, \underline{F})$ we have $\forall i \in I, X=\underline{F}(X)=\bigcap_{j \in I} F_{j}(X) \subseteq$ $F_{i}(X)$ and dually $F_{i}(X) \subseteq \bigcup_{j \in I} F_{j}(X)=\bar{F}(X)=X$ so that by antisymmetry $X=F_{i}(X)$ and $f p(\bar{F}, \underline{F}) \subseteq f p\left(\left\{F_{i}: i \in I\right\}\right)$. By antisymmetry $f p(\bar{F}, \underline{F})=f p\left(\left\{F_{i}: i \in I\right\}\right)$ so that by Lemma $6.1, f p\left(\left\{F_{i}: i \in I\right\}\right)$

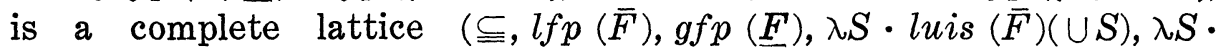
llis $(\underline{F})(\cap S))$.

COROLlaRY 6.3. Let $D$ be an arbitrary element of $L$, then luis $\left(\lambda Z \cdot \bigcup_{i \in I} F_{i}(Z)\right) \circ$ llis $\left(\lambda Z \cdot Z \cap\left(\bigcap_{i \in I} F_{i}(Z)\right)\right)(D)$ and llis $(\lambda Z$. $\left.\bigcap_{i \in I} F_{i}(Z)\right) \circ$ luis $\left(\lambda Z \cdot Z \cup\left(\bigcup_{i \in I} F_{i}(Z)\right)\right)(D)$ are common fixed points of the $F_{i}, i \in I$ which are greater than or equal to any common fixed point of the $F_{i}$ less than or equal to $D$ and which are less than or 
equal to any common fixed point of the $F_{i}$ greater than or equal to D. Moreover

$$
\begin{aligned}
\operatorname{luis} & \left(\lambda Z \cdot \bigcup_{i \in I} F_{i}(Z)\right) \circ \operatorname{llis}\left(\lambda Z \cdot Z \cap\left(\bigcap_{i \in I} F_{i}(Z)\right)\right)(D) \\
& \cong \operatorname{lis}\left(\lambda Z \cdot \bigcap_{i \in I} F_{i}(Z)\right) \circ \operatorname{luis}\left(\lambda Z \cdot Z \cup\left(\bigcup_{i \in I} F_{i}(Z)\right)\right)(D) .
\end{aligned}
$$

CoROLlary 6.4. The set $f p\left(\left\{F_{i}: i \in I\right\}\right)$ of common fixed points of the family $\left\{F_{i}: i \in I\right\}$ is the image of $L$ by the lower preclosure operator luis $\left(\lambda Z \cdot \bigcup_{i \in I} F_{i}(Z)\right) \circ$ llis $\left(\lambda Z \cdot Z \cap\left(\bigcap_{i \in I} F_{i}(Z)\right)\right)$ and the image of $L$ by the upper preclosure operator llis $\left.\lambda Z \cdot \bigcap_{i \in I} F_{i}(Z)\right)$ 。 luis $\left(\lambda Z \cdot Z \cup\left(\bigcup_{i \in I} F_{i}(Z)\right)\right)$.

Let $\left\{F_{i}: i \in I\right\}$ be a finite family of monotone commuting operators on the complete lattice $L$ into itself. If we assume that $I$ is well-ordered (i.e., $I=\left\{i_{\alpha}: \alpha \leqq \gamma\right\}$ where $\gamma \in \omega$ ) then we denote $\lambda Z \cdot F_{i_{0}}\left(F_{i_{1}}\left(\cdots F_{i_{\gamma}}(Z) \cdots\right)\right)$ by $\bigcirc_{i \in I} F_{i}$.

Applying Theorem 5.1 to $\bigcirc_{i \in I} F_{i}$ and Theorem 6.2 to $\left\{F_{i}: i \in I\right\}$ a natural question is whether $f p\left(\bigcirc_{i \in I} F_{i}\right)=f p\left(\left\{F_{i}: i \in I\right\}\right)$. The answer is affirmative thanks to the following:

THEOREM 6.5 .

$$
\begin{array}{r}
\operatorname{luis}\left(\lambda Z \cdot \bigcup_{i \in I} F_{i}(Z)\right) \circ \operatorname{llis}\left(\lambda Z \cdot Z \cap\left(\bigcap_{i \in I} F_{i}(Z)\right)\right) \\
=\operatorname{luis}\left(\bigcirc_{i \in I} F_{i}\right) \circ \operatorname{llis}\left(\lambda Z \cdot Z \cap\left(\bigcirc_{i \in I} F_{i}\right)(Z)\right) \\
\operatorname{llis}\left(\lambda Z \cdot \bigcap_{i \in I} F_{i}(Z)\right) \circ \operatorname{luis}\left(\lambda Z \cdot Z \cup\left(\bigcup_{i \in I} F_{i}(Z)\right)\right) \\
=\operatorname{llis}\left(\bigcirc_{i \in I} F_{i}\right) \circ \operatorname{luis}\left(\lambda Z \circ Z \cup\left(\bigcirc_{i \in I} F_{i}(Z)\right)\right) .
\end{array}
$$

Proof. It is sufficient to prove that if $D$ is a prefixed point of each $F_{i}$ such that $i \in I$ then luis $\left(\lambda Z \cdot \bigcup_{i \in I} F_{i}(Z)\right)(D)=\operatorname{luis}\left(\bigcirc_{i \in I} F_{i}\right)(D)$. Since $\left\{\forall i \in I, D \subseteq F_{i}(D)\right\}$ we have by monotony and the commuting property $D \subseteq\left(\bigcirc_{i \in I} F_{i}\right)(D)$ and Theorem 3.3 implies that $P=$ luis $\left(\bigcirc_{i \in I} F_{i}\right)(D)=\left(\bigcirc_{i \in I} F_{i}\right)(P)$ and $D \subseteq P$. For every $j \in I$ we have $D \subseteq F_{j}(D) \subseteq F_{j}(P)=F_{j}\left(\left(\bigcirc_{i \in I} F_{i}\right)(P)\right)=\left(\bigcirc_{i \in I} F_{i}\right)\left(F_{j}(P)\right)$. Therefore $F_{j}(P)$ is a fixed point of $\bigcirc_{i \in I} F_{i}$ greater than or equal to $D$ so that by Theorem $3.3\left\{\forall j \in I, P \subseteq F_{j}(P)\right\}$. Then by monotony and transitivity $P \subseteq F_{i_{\gamma}}(P) \subseteq F_{i_{1}}\left(\cdots F_{i_{\gamma}}(P) \cdots\right) \subseteq\left(\bigcirc_{i \in I} F_{i}\right)(P)=P$ so that $\forall j \in I, P=$ $F_{j}(P) . \quad P$ is a common fixed point of the family $\left\{F_{i}: i \in I\right\}$ greater than or equal to $D$. Let $Q$ be another common fixed point of $\left\{F_{i}: i \in I\right\}$ greater than or equal to $D$. Then $\left(\bigcirc_{i \in I} F_{i}\right)(Q)=Q$ so that by Theorem 3.3 we have $P \subseteq Q$. Hence $P$ is the least common fixed point of the family $\left\{F_{i}: i \in I\right\}$ greater than or equal to $D$. 
By Corollary 6.3, $R=\operatorname{luis}\left(\lambda Z \cdot \bigcup_{i \in I} F_{i}(Z)\right)(D)$ is a common fixed point of $\left\{F_{i}: i \in I\right\}$ greater than or equal to $D$. Let $Q$ be another common fixed point of $\left\{F_{\imath}: i \in I\right\}$ greater than or equal to $D$. Then $\bigcup_{i \in I} F_{i}(Q)=Q$ so that by Theorem 3.3 we have $R \subseteq Q$. Hence $R$ is the least common fixed point of the family $\left\{F_{i}: i \in I\right\}$ greater than or equal to $D$.

By existence and unicity of the least common fixed point of the family $\left\{F_{1}: i \in I\right\}$ greater than or equal to $D$, we conclude $\operatorname{luis}\left(\bigcirc_{i \in I} F_{i}\right)(D)=P=R=\operatorname{luis}\left(\lambda Z \cdot \bigcup_{i \in I} F_{i}(Z)(D)\right.$.

7. Fixed point theorems for continuous operators. An operator $F$ on the complete lattice $L$ into itself is upper-semi-continuous if and only if for every ordinal $\delta \leqq \omega$ and every $\delta$-termed increasing chain $\left\langle C^{\alpha}, \alpha \in \delta\right\rangle$ of elements of $L$ we have $F\left(\bigcup_{\alpha \in \delta} C^{\alpha}\right)=$ $\mathrm{U}_{\alpha \in \delta} F\left(C^{\alpha}\right)$. The dual notion is the one of lower-semi-continuous operator. An operator is continuous when it is lower and uppersemi-continuous.

Since semi-continuity implies monotony the results of paragraphs 3,4 , and 5 can be applied to continuous operators. However the proofs are simplified since one can consider $(\omega+1)$-termed iteration sequences. For example, Theorem 3.3 can be reformulated as follows:

THEOREM 7.1. Let $F$ be an upper-semi-continuous operator on the complete lattice $L$ into itself. An upper iteration sequence $\left\langle X^{\delta}, \delta \in \min (\mu, \omega+1)\right\rangle$ for $F$ starting with $D \in$ prefp $(F)$ is a stationary increasing chain, its limit luis $(F)(D)$ is the least of the fixed points of $F$ greater than or equal to $D$.

Proof. When $\mu>\omega+1$ Definition 2.1, Theorem 3.3 and uppersemi-continuity imply $X^{\omega+1}=F\left(X^{\omega}\right)=F\left(\bigcup_{\alpha<\omega} X^{\alpha}\right)=\bigcup_{\alpha<\omega} F\left(X^{\alpha}\right)=$ $\mathrm{U}_{\alpha<\omega} X^{\alpha+1} \subseteq \mathrm{U}_{\alpha<\omega} X^{\alpha}=X^{\omega}$. Also by Theorem 3.3, $X^{\omega} \cong X^{\omega+1}$ so that by antisymmetry $X^{\omega}=X^{\omega+1}$. Then by transfinite induction it is easy to show that $\left\{\forall \beta: \omega \leqq \beta<\mu, X^{\omega}=X^{\beta}\right\}$.

When considering a family of commuting monotone operators the results of paragraph 6 can be perfected as follows:

LemMa 7.2. Let $\underline{F}$ and $\bar{F}$ be upper-semi-continuous operators on the complete lattice $L$ into itself such that $\bar{F} \circ \underline{F} \cong \underline{F} \circ \bar{F}$ and $\underline{F} \subseteq \bar{F}$. Then for every prefixed point $D$ of $F$ we have:

$$
\{\underline{F}(D)=\bar{F}(D)\} \Longrightarrow\{\operatorname{luis}(\underline{F})(D)=\operatorname{luis}(\bar{F})(D)\} .
$$


Proof. Let $\left\langle X^{\delta}, \delta \in \min (\omega+1, \mu)\right\rangle$ and $\left\langle Y^{\delta}, \delta \in \min (\omega+1, \mu)\right\rangle$ be respectively the upper iteration sequences for $\underline{F}$ and $\bar{F}$ starting with the prefixed point $D$ of $\underline{F}$ and $\bar{F}$.

For $\delta=0$ we know by hypothesis and 2.1(a) that $D=X^{0}=Y^{0} \subseteq$ $\bar{F}\left(X^{0}\right)=\underline{F}\left(Y^{0}\right)$.

Assume that $\delta \in \min (\omega+1, \mu)$ is a successor ordinal such that $X^{\delta-1}=Y^{\delta-1}$ and $\bar{F}\left(X^{\delta-1}\right)=\underline{F}\left(Y^{\delta-1}\right)$. Then by $2.1(\mathrm{~b}) X^{\delta}=\underline{F}\left(X^{\delta-1}\right)=$ $\underline{F}\left(Y^{\delta-1}\right)$ and $\bar{F}\left(X^{\delta-1}\right)=\bar{F}\left(Y^{\delta-1}\right)=Y^{\delta}$ so that by induction hypothesis and transitivity $X^{\delta}=Y^{\delta}$. Also since $\underline{F} \subseteq \bar{F}$ and $X^{\delta}=Y^{\delta}$ we know that $\underline{F}\left(Y^{\delta}\right) \subseteq \bar{F}\left(X^{\delta}\right)$. Since $\bar{F} \circ \underline{F} \subseteq \underline{F} \circ \bar{F}$ and $X^{\delta-1}=Y^{\delta-1}$ we know that $\bar{F}\left(\underline{F}\left(X^{\delta-1}\right)\right) \subseteq \underline{F}\left(\bar{F}\left(Y^{s-1}\right)\right)$ so that by Definition $2.1(\mathrm{~b})$ we get $\bar{F}\left(X^{\delta}\right) \subseteq$ $\underline{F}\left(Y^{\delta}\right)$. By antisymmetry we conclude $\bar{F}\left(X^{\delta}\right)=\underline{F}\left(Y^{\delta}\right)$.

Assume that $\delta \in \min (\omega+1, \mu)$ is a limit ordinal then $\delta=\omega$. If by induction hypothesis $\left\{\forall \beta<\omega, X^{\beta}=Y^{\beta}\right.$ and $\bar{F}\left(X^{\beta}\right)=\underline{F}\left(Y^{\beta}\right)$ then by 2.1(c) and definition of least upper bounds we have $X^{\omega}=$ $\mathrm{U}_{\alpha<\omega} X^{\alpha}=\mathrm{U}_{\alpha<\omega} Y^{\alpha}=Y^{\omega}$. The same way by upper-semi-continuity, $\bar{F}\left(X^{\omega}\right)=\bar{F}\left(\bigcup_{\alpha<\omega} X^{\alpha}\right)=\bigcup_{\alpha<\omega} \bar{F}\left(X^{\alpha}\right)=\bigcup_{\alpha<\omega} \underline{F}\left(Y^{\alpha}\right)=\underline{F}\left(\bigcup_{\alpha<\omega} Y^{\alpha}\right)=\underline{F}\left(Y^{\omega}\right)$.

By transfinite induction and Theorem 7.1 we conclude luis $(\underline{\boldsymbol{F}})(D)=\operatorname{luis}(\bar{F})(D)$.

As an application of Lemma 7.2 for $D=\perp$, we get:

THEOREM 7.3. Let $\left\{F_{i}: i \in I\right\}$ be a family of commuting operators on the complete lattice $L$ into itself. Then $\left\{\left\{\forall i \in I, F_{i}\right.\right.$ is upper-semicontinuous $\}$ and $\left.\left\{\forall i, j \in I, F_{i}(\perp)=F_{j}(\perp)\right\}\right\} \Rightarrow\left\{\forall i, j \in I, \operatorname{lfp}\left(F_{i}\right)=\right.$ $\left.l f p\left(F_{j}\right)\right\}$.

8. Remark. In our proofs it is the existence of lower or upper bounds of chains and not the existence of lower or upper bounds of arbitrary sets that is crucial. The same remark was made by numerous authors who generalized Tarski's fixed point theorem to weaken the completeness hypothesis (see among others Abian and Brown [1], Höft [9], Pasini [15], Pelczar [16], Markowsky [14], Ward [20], Wolk [22]). This was also the case for Tarski's fixed point theorem on commuting maps (see a.o., DeMarr [6], Markowsky [14], Pelczar [17], Smithson [18], Wong [23]). Along the same lines our results could be strengthened to be applicable to partially ordered sets which are not complete lattices.

ACKNOWLEDGMENT. The authors thank the referee in particular for strengthening the initial version of Theorem 4.1. 


\section{REFERENCES}

1. S. Abian and A. B. Brown, A theorem on partially ordered sets with applications to fixed point theorems, Canad. J. Math., 13 (1961), 78-82.

2. H. Amann, Fixed point equations and non-linear eigenvalue problems in ordered Banach spaces, SIAM Review 18, 4 (October 1976), 620-709.

3. G. Birkhoff, Lattice theory, Amer. Math. Soc. Colloquium Publications 25, 3rd ed., Providence, Rhode Island, (1976).

4. A. Church, The calculi of lambda-conversion, Annals of Math. Studies, 6 (1951), Princeton University Press, Princeton, N.J.

5. P. Cousot and R. Cousot, Abstract interpretation: a unified lattice model for static analysis of programs by construction or approximation of fixpoints, Conf. Rec. of the Fourth ACM Symp. on Principles of Programming Languages, Los Angeles, Calif. (Jan. 1977), 238-252.

6. R. DeMarr, Common fixed points for isotone mappings, Colloquium Math., 13 (1964), $45-48$.

7. V. Devidé, On monotonous mappings of complete lattices, Fundamenta Mathematicae, LIII (1964), 147-154.

8. P. Hitchcock and D. Park, Induction rules and proofs of termination, Proc. of a Symp. on Automata, Languages and Programming, North-Holland Pub. Co., Amsterdam, (1973), 225-251.

9. H. Höft and M. Höft, Some fixed point theorems for partially ordered sets, Canad. J. Math., 5 (1976), 992-997.

10. S. C. Kleene, Introduction to metamathematics, North-Holland Pub. Co., Amsterdam, (1952).

11. I. I. Kolodner, On completeness of partially ordered sets and fixpoints theorems for isotone mappings, Amer. Math. Monthly, 75 (1968), 48-49.

12. Y. Ladegaillerie, Prefermeture sur un ensemble ordonné, RAIRO, 1 (Avril 1973), 35-43.

13. Z. Manna and A. Shamir, The convergence of functions to fixed points of recursive definitions, Theoretical Computer Science, 6 (1978), 109-141.

14. G. Markowsky, Chain-complete posets and directed sets with applications, Algebra Univ., 6 (1976), 53-58, Birkhaüser Verlag, Basel.

15. A. Pasini, Some fixed point theorems of the mappings of partially ordered sets, Rend. Sem. Mat. Univ. Padova, 51 (1974), 167-177.

16. A. Pelczar, On the invariant points of a transformation, Ann. Polon. Math., 11 (1961), 199-202.

17. - Remarks on commutings mappings in partially ordered spaces, Zeszyty Nauk. Univ. Jagiello., Prace Mat. Zeszyt, 15 (1971), 131-133.

18. R. E. Smithson, Fixed points in partially ordered sets, Pacific J. Math., 1 (1973), 363-367.

19. A. Tarski, A lattice theoretical fixpoint theorem and its applications, Pacific J. Math., 5 (1955), 285-310.

20. L. E. Ward Jr., Completeness in semi-lattices, Canad. J. Math., 9 (1957), 578-582.

21. M. Ward, The closure operators of a lattice, Annals Math., 43 (1942), 191-196.

22. E. S. Wolk, Dedekind completeness and a fixed point theorem, Canad. J. Math., 9 (1957), 400-405.

23. J. S. W. Wong, Common fixed points of commuting monotone mapping, Canad. J. Math., 19 (1967), 617-620.

Received December 5, 1977 and in revised form June 26, 1978. Work of the first author was supported in part by CNRS, Laboratoire associé $n^{\circ} 7$ and ATP-Informatique D3119 and the second author in part by IRIA-SESORI under grant 76160 .

LABORATOIRE I.M.A.G.

U. S. M. G., BP. 53X

38041 Grenoble Cedex, France 



\section{PACIFIC JOURNAL OF MATHEMATICS}

\section{EDITORS}

DoNALD BABBITT (Managing Editor)

University of California

Los Angeles, California 90024

HUGo Rossi

University of Utah

Salt Lake City, UT 84112

C. C. MOORE and ANDREW OGG

University of California

Berkeley, CA 94720
J. DUGUNDJI

Department of Mathematics University of Southern Californı Los Angeles, California 90007

R. Finn and J. Milgram Stanford University Stanford, California 94305

\section{ASSOCIATE EDITORS}

E. F. BECKENBACH

B. H. Neumann
F. WOLF

K. YOSHIDA

\section{SUPPORTING INSTITUTIONS}

UNIVERSITY OF BRITISH COLUMBIA CALIFORNIA INSTITUTE OF TECHNOLOGY UNIVERSITY OF CALIFORNIA MONTANA STATE UNIVERSITY UNIVERSITY OF NEVADA, RENO NEW MEXICO STATE UNIVERSITY OREGON STATE UNIVERSITY UNIVERSITY OF OREGON
UNIVERSITY OF SOUTHERN CALIFORNIA STANFORD UNIVERSITY UNIVERSITY OF HAWAII UNIVERSITY OF TOKYO UNIVERSITY OF UTAH WASHINGTON STATE UNIVERSITY UNIVERSITY OF WASHINGTON 


\section{Pacific Journal of Mathematics}

Vol. 82, No. $1 \quad$ January, 1979

Werner Bäni, Subspaces of positive definite inner product spaces of countable dimension ...................................... 1

Marilyn Breen, The dimension of the kernel of a planar set..............

Kenneth Alfred Byrd, Right self-injective rings whose essential right ideals

are two-sided

Patrick Cousot and Radhia Cousot, Constructive versions of Tarski's fixed

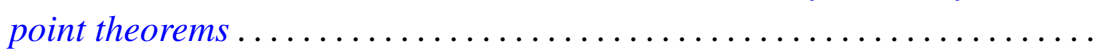

Ralph S. Freese, William A. Lampe and Walter Fuller Taylor, Congruence lattices of algebras of fixed similarity type. $I \ldots \ldots \ldots \ldots \ldots \ldots \ldots$

Cameron Gordon and Richard A. Litherland, On a theorem of Murasugi .....

Mauricio A. Gutiérrez, Concordance and homotopy. I. Fundamental

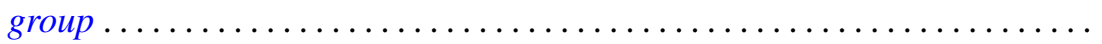

Richard I. Hartley, Metabelian representations of knot groups .............

Ted Hurley, Intersections of terms of polycentral series of free groups and free

Lie algebras ........................................

Roy Andrew Johnson, Some relationships between measures ............ 117

Oldřich Kowalski, On unitary automorphisms of solvable Lie algebras .......

Kee Yuen Lam, $K O$-equivalences and existence of nonsingular bilinear

maps...................................................

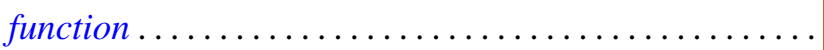

Robert A. Messer and Alden H. Wright, Embedding open 3-manifolds in compact 3-manifolds ............................

Gerald Ira Myerson, A combinatorial problem in finite fields. I . .

James Nelson, Jr. and Mohan S. Putcha, Word equations in a band of paths.

Baburao Govindrao Pachpatte and S. M. Singare, Discrete generalized Gronwall inequalities in three independent variables . .

William Lindall Paschke and Norberto Salinas, $C^{*}$-algebras associated with free products of groups ........................

Bruce Reznick, Banach spaces with polynomial norms ....

David Rusin, What is the probability that two elements of a finite group

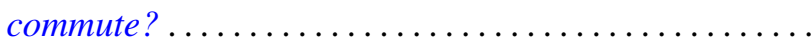

M. Shafii-Mousavi and Zbigniew Zielezny, On hypoelliptic differential operators of constant strength ...

Joseph Gail Stampfli, On selfadjoint derivation ranges .... . . .

Robert Charles Thompson, The case of equality in the matrix-valued triangle

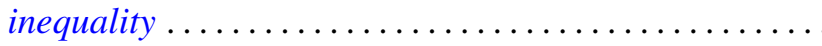

Marie Angela Vitulli, The obstruction of the formal moduli space in the negatively graded case. 